\title{
Empowering community dwelling older citizens to improve their balance with a novel technology platform
}

\author{
Dimitrios Gatsios $^{1}$, Doris Eva Bamiou ${ }^{2}$, Sergi Costafreda ${ }^{2}$, Eleni. I. Georga ${ }^{1}$, Konstan- \\ tina K. Kourou ${ }^{1}$, Themis Exarchos ${ }^{3}$, Kostas M. Tsiouris ${ }^{1,4}$, Dimitrios I. Fotiadis ${ }^{1,5}$ \\ ${ }^{1}$ Unit of Medical Technology and Intelligent Information Systems, Department of Materials \\ Science and Engineering, University of Ioannina, GR45110, Ioannina, Greece \\ ${ }^{2}$ UCL Ear Institute \& University College London Hospitals Biomedical Research Centre \\ Hearing \& Deafness, WC1X 8EE, London, United Kingdom \\ ${ }^{3}$ Department of Informatics, Ionian University, GR49100, Corfu, Greece \\ ${ }^{4}$ School of Electrical and Computer Engineering, National Technical University of Athens, \\ GR15773, Athens, Greece \\ ${ }^{5}$ Dept. of Biomedical Research, IMBB-FORTH, GR45110, Ioannina, Greece \\ fotiadis@cc.uoi.gr
}

\begin{abstract}
The prevalence of balance deficits increases as the population is ageing. Such deficits are associated with the increased incidence of falls which in turn is linked with substantial limited functionality and morbidity. Vestibular rehabilitation therapy (VRT) as a component of the treatment has been shown to be effective in reducing symptoms and improving balance. HOLOBALANCE is an intervention based on a novel technology platform for providing VRT unsupervised, at home which means that motivating citizens to be compliant and promoting empowerment are the cornerstones for its wide adoption. Here we present how citizens empowerment is being addressed in HOLOBALANCE.
\end{abstract}

Keywords: Empowerment, vestibular rehabilitation therapy, human machine interaction.

\section{Introduction}

As population ages, an increasing number of community dwelling citizens face permanent balance problems that lead to limited functionality. For these citizens, dizziness, which is a significant risk factor for falls [1], and/or imbalance are the most frequent reason for visiting a physician [2]. Falls are a leading cause of serious injury. The overall rate of older adult deaths from falls increased 31\% from 2007 to 2016 (3.0\% per year) [3]. In a recent study [4] the estimated medical costs attributable to fatal and nonfatal falls was approximately $\$ 50.0$ billion and the overall medical spending for fatal falls was estimated to be $\$ 754$ million. It is estimated that peripheral and central vestibular deficits cause around $50 \%$ of the cases of dizziness. 
Vestibular rehabilitation therapy (VRT) is a form of physiotherapy that aims to retrain postural, movement and sensory strategies and to adapt these to changing contexts by means of a range of gaze, standing, and gait exercises [5]. In recent trials [6] and studies [7] VRT has been shown to be more effective than generic exercises in resolving symptoms after vestibular impairment [8]. VRT is predominantly delivered at home, daily, without supervision. It is personalized, defined and directed by expert physiotherapists and it is largely dependent on patient engagement and motivation, since the number of clinical visits is very limited and varies among settings. The exercises are progressive with increasing difficulty as physiological compensation (i.e. the recovery process that harnesses the adaptive, plastic properties of the posture and balance control system) and symptom habituation occur. Due to the nature of the intervention and as literature confirms [9-11] empowerment is of outmost importance for keeping the elderly individuals engaged. Citizen and patient empowerment is one of the strategic directions and priority areas for European policy action for health and well-being up to 2020 according to WHO [12] which actively and continuously promotes it.

\section{The concept of empowerment in HOLOBALANCE}

Empowerment is a key concept when developing health promotion technological solutions and strategies for community dwelling citizens. In the context of balance disorders, empowerment can be achieved by providing expert guided vestibular rehabilitation at home, that is to a large extent informed by citizen choice (in terms of rehabilitation goals, types of exercise and activities, timing of these etc.) and different tools to support adoption of a more active lifestyle. This is made possible and provided via a novel technological platform.

The HOLOBALANCE platform among other functionalities, offers a range of choices to the patient and enables clinicians to monitor their patients and their behaviors during the different interventions employed in it in order to understand their patients' engagement and willingness for change. As evidence suggests, shared decision-making [14-16], rewarding [17], goal setting [18], action planning [19] and selfengagement [20], are the other core elements for achieving empowerment. All of these can be facilitated by technology.

The design of the system is user-centric and based on direct feedback from older users [21] and the software development approach is agile in order to iteratively include that feedback. The HOLOBALANCE platform makes use of the Internet of Things (IoT) and edge computing for monitoring and evaluating in real time the performance of the users during physiotherapy exercises, exergames and cognitive training tasks. Personalized and adaptive coaching is provided to the users during the daily sessions, by employing progressive and reinforcement learning methods. Data are collected in the cloud backend for: a) exercise performance and adherence using processing of sensor data and analytic methods, b) stress and frustration using machine learning algorithms, c) active and sedentary periods and steps, distance etc. using the Fitbit backend, and d) symptoms related to the exercise performance during the reha- 
bilitation regime. These data are made available through a Dashboard to the physiotherapists, thus facilitating the remote monitoring of patients' progress in the rehabilitation program and supporting the physiotherapist decision making for adjustments and progression in the management plan.

The novel human machine interaction (HMI) consists of a hologram acting as a physiotherapist surrogate, who guides older users with balance disorders through the whole multisensory balance rehabilitation regime. Holograms aim to take HMI a step beyond the current state of the art [22]. Augmented reality exergames consist of virtual objects and tasks presented in a mixed reality environment to provide stimuli to the users. Such interventions are gaining interest and are effective [23] in reducing falls. Both holograms and exergames are projected through a head mounted device with a smartphone embedded on it, which is a solution with reduced costs, compared to similar mixed reality headsets, and increased accessibility and usability. A mobile app is also developed for remote auditory training aiming to improve speech in noise perception and auditory memory in accord with recent studies [24].

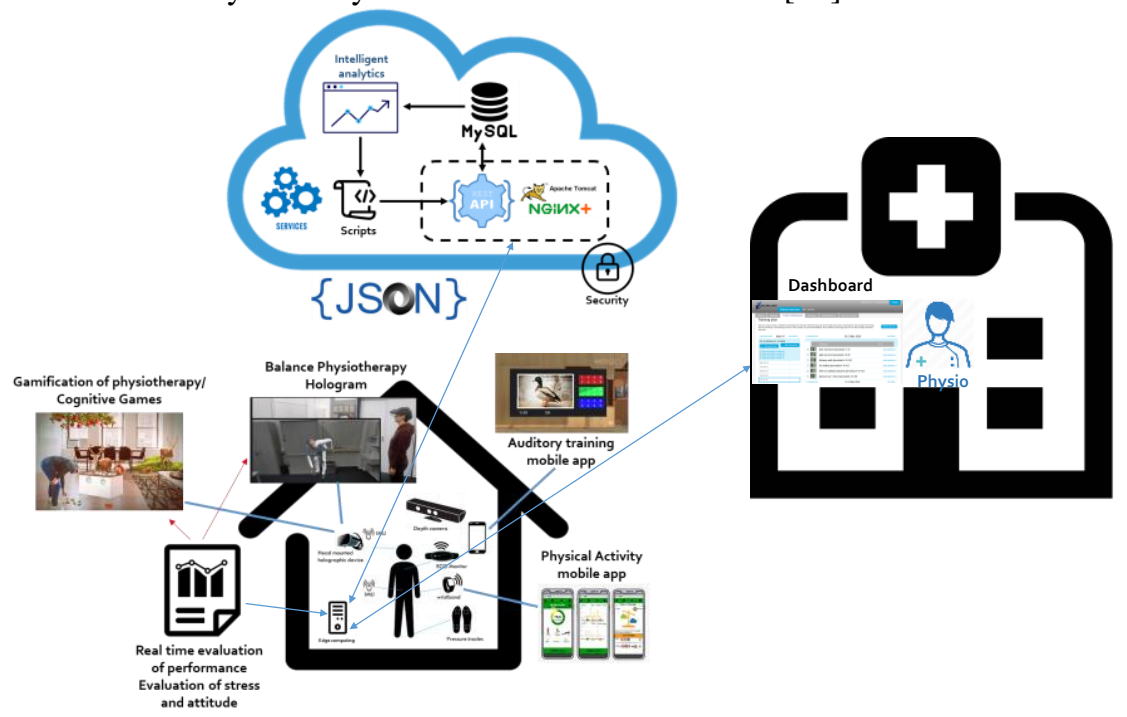

Fig. 1. The HOLOBALANCE conceptual architecture.

HOLOBALANCE adopts a holistic approach that aims to empower citizens. In addition to the intervention related empowering features, it provides citizens with access and control over their data, since this is one of the key elements of empowerment, as also emphasized in GDPR too [25]. Data download on demand, tagging and tracking personal data, transparency over data usage and permissions including the right to revoke are all included in this regulation.

HOLOBALANCE is personalized, in that the vestibular rehabilitation therapy plan and the activity goals will be co-decided by the patients and the physiotherapists at baseline and will be revised in follow up visits every 4 weeks. In between visits the physiotherapist will revise the weekly plan based on the data from the platform about 
performance of exercises, stress levels, compliance, progress in activity goals etc. against automated suggestions coming from reinforcement learning methods.

The HOLOBALANCE approach is based on the Capability, Opportunity and Motivation (COM) and Behavior (B) theoretical model in order to systematically identify the sources of the behavior that should be targeted for intervention and propose appropriate and specific Information Technology components (holograms, exergames, cognitive games, mobile apps) that provide the different intervention strands (balance physiotherapy, physical activity, cognitive and auditory training) to the users in order to achieve motivation and compliance to the home based rehabilitation.

HOLOBALANCE further aims to educate the patient by providing knowledge and information thus promoting health literacy, i.e. one's knowledge about his own health status and the available options to manage, since these also are core elements of empowerment. This element is achieved by educating the patient during the baseline and follow up visits, and with phone calls whenever it is needed, e.g. if the patient is not adherent to the program. Additionally, knowledge about balance disorders as well as about treatment options will be available in the patients' mobile app (videos, written materials, graphs) that will provide a continuous and reliable source of information. Finally, this will be achieved within the patient e-forum and virtual community, since patients tend to exchange information and tips in these.

The platform will promote engagement and enjoyment with feedback, challenge and rewarding mechanisms that will be embedded in all technological components. The hologram instructs the user, shows him the correct performance of the exercises and provides feedback for correcting him as well as rewards dependent on the performance to motivate him. The exergames provide feedback and rewards in a similar to the hologram way. Progress of auditory and cognitive training is also monitored and evaluated for the provision of personalized feedback. Self-monitoring is enabled through the mobile app in which all rewards and achievements are recorded and available. It also enables action planning and goal setting. The users can set their specific activity goals, mainly for steps and distance covered, as well as goals co-decided with their clinicians. Such shared decision making is nowadays central to evidencebased medicine and it is core component of patient-centered care. Patient empowerment is key to shared decision-making which can only happen when a patient admits responsibility for his health.

\section{Conclusions}

Current theoretical models and scientific evidence indicate that patient empowerment for patients with balance disorders at risk of falls can be achieved by adopting a holistic approach, to provide personalized home based, unsupervised interventions, by employing advanced technology and novel human machine interactions. Health literacy is the basis of such intervention since it enables citizens to use their own care information and information about balance problems in order to motivate them to engage with and complete their treatment plan. Feedback, goal setting, rewarding and 
action planning are other core elements of this intervention. Measuring empowerment within HOLOBALANCE, which is a challenging task [26], will add evidence to the field and will be one of the outcomes of the multicenter, randomized controlled trial that will be conducted towards the end of this project to assess the feasibility and estimate the efficacy of the whole concept.

\section{References}

1. Agrawal, Y., Carey, J. P., Della Santina, C. C., Schubert, M. C., \& Minor, L. B: Disorders of balance and vestibular function in US adults: data from the National Health and Nutrition Examination Survey, 2001-2004. Archives of internal medicine 169(10), 938-944 (2009).

2. Neuhauser, H. K., Radtke, A., Von Brevern, M., Feldmann, M., Lezius, F., Ziese, T., \& Lempert, T.: Migrainous vertigo: prevalence and impact on quality of life. Neurology, 67(6), 1028-1033 (2006).

3. Burns, E., \& Kakara, R.: Deaths from falls among persons aged $\geq 65$ years-United States, 2007-2016. Morbidity and Mortality Weekly Report, 67(18), 509 (2018).

4. Florence, C. S., Bergen, G., Atherly, A., Burns, E., Stevens, J., \& Drake, C.: Medical costs of fatal and nonfatal falls in older adults. Journal of the American Geriatrics Society, 66(4), 693-698 (2018).

5. Wrisley, D. M., \& Pavlou, M.: Physical therapy for balance disorders. Neurologic clinics, 23(3), 855-874 (2005).

6. Rossi-Izquierdo, M., Gayoso-Diz, P., Santos-Pérez, S., Del-Río-Valeiras, M., FaraldoGarcía, A., Vaamonde-Sánchez-Andrade, I., ... \& Soto-Varela, A.: Vestibular rehabilitation in elderly patients with postural instability: reducing the number of falls - a randomized clinical trial. Aging clinical and experimental research, 30(11), 1353-1361 (2018).

7. Kundakci, B., Sultana, A., Taylor, A. J., \& Alshehri, M. A.: The effectiveness of exercisebased vestibular rehabilitation in adult patients with chronic dizziness: A systematic review. F1000Research, 7 (2018).

8. McDonnell, M. N., \& Hillier, S. L.: Vestibular rehabilitation for unilateral peripheral vestibular dysfunction. Cochrane database of systematic reviews, (1) (2015).

9. Madsen, W., Ambrens, M., \& Ohl, M.: Enhancing resilience in community-dwelling older adults: a rapid review of the evidence and implications for public health practitioners. Frontiers in public health, 7;7:14 (2019).

10. Seah, B., Kowitlawakul, Y., Jiang, Y., Ang, E., Chokkanathan, S., \& Wang, W.: A review on healthy ageing interventions addressing physical, mental and social health of independent community-dwelling older adults. Geriatric Nursing, 40(1), 37-50 (2018).

11. Werbrouck, A., Swinnen, E., Kerckhofs, E., Buyl, R., Beckwée, D., \& De Wit, L.: How to empower patients? A systematic review and meta-analysis. Translational behavioral medicine, 8(5), 660-674 (2018).

12. World Health Organization. Regional Office for Europe. Health 2020 policy framework and strategy document [Internet]. Geneva: WHO; 2012 [cited 2015 Feb. 28].

13. Kouris, I., Sarafidis, M., Androutsou, T., \& Koutsouris, D.: HOLOBALANCE: An Augmented Reality virtual trainer solution for balance training and fall prevention. In: 2018 40th Annual International Conference of the IEEE Engineering in Medicine and Biology Society (EMBC), pp. 4233-4236, IEEE. Honolulu, Hawaii (2018). 
14. Small, N., Bower, P., Chew-Graham, C. A., Whalley, D., \& Protheroe, J.: Patient empowerment in long-term conditions: development and preliminary testing of a new measure. BMC Health Services Research, 13(1), 263 (2013).

15. Fotoukian, Z., Shahboulaghi, F. M., Khoshknab, M. F., \& Mohammadi, E.: Concept analysis of empowerment in old people with chronic diseases using a hybrid model. Asian Nursing Research, 8(2), 118-127 (2014).

16. Salmon, P., \& Hall, G. M.: Patient empowerment or the emperor's new clothes. Journal of the Royal Society of Medicine, 97(2), 53-56 (2004).

17. Allam, A., Kostova, Z., Nakamoto, K., \& Schulz, P. J.: The effect of social support features and gamification on a Web-based intervention for rheumatoid arthritis patients: randomized controlled trial. Journal of medical Internet research, 17(1) (2015).

18. Young, H., Miyamoto, S., Ward, D., Dharmar, M., Tang-Feldman, Y., \& Berglund, L.: Sustained effects of a nurse coaching intervention via telehealth to improve health behavior change in diabetes. Telemedicine and e-Health, 20(9), 828-834 (2014).

19. Riva, S., Camerini, A. L., Allam, A., \& Schulz, P. J.: Interactive sections of an Internetbased intervention increase empowerment of chronic back pain patients: randomized controlled trial. Journal of medical Internet research, 16(8) (2014).

20. McCarrier, K. P., Ralston, J. D., Hirsch, I. B., Lewis, G., Martin, D. P., Zimmerman, F. J., \& Goldberg, H. I.: Web-based collaborative care for type 1 diabetes: a pilot randomized trial. Diabetes technology \& therapeutics, 11(4), 211-217 (2009).

21. Mostajeran, F., Katzakis, N., Ariza, O., Freiwald, J. P., \& Steinicke, F.: Welcoming a Holographic Virtual Coach for Balance Training at Home: Two Focus Groups with Older Adults. In: Proceedings of 26th IEEE Conference on Virtual Reality and 3D User Interfaces, ahead of publish, IEEE, Osaka (2019).

22. de Amorim, J. S. C., Leite, R. C., Brizola, R., \& Yonamine, C. Y.: Virtual reality therapy for rehabilitation of balance in the elderly: a systematic review and META-analysis. Advances in Rheumatology, 58(1), 18 (2018).

23. Stanmore, E. K., Mavroeidi, A., de Jong, L. D., Skelton, D. A., Sutton, C. J., Benedetto, V., L/ A. Munford, W. Meekes, V. Bell \& Todd, C.: The effectiveness and costeffectiveness of strength and balance Exergames to reduce falls risk for people aged 55 years and older in UK assisted living facilities: a multi-centre, cluster randomised controlled trial. BMC medicine, 17(1), 49 (2019).

24. Abrams, H. B., Bock, K., \& Irey, R. L.: Can a remotely delivered auditory training program improve speech-in-noise understanding? American journal of audiology, 24(3), 333337 (2015).

25. De Hert, P., Papakonstantinou, V., Malgieri, G., Beslay, L., \& Sanchez, I.: The right to data portability in the GDPR: Towards user-centric interoperability of digital services. Computer Law \& Security Review, 34(2), 193-203 (2018).

26. Barr, P. J., Scholl, I., Bravo, P., Faber, M. J., Elwyn, G., \& McAllister, M.: Assessment of patient empowerment-a systematic review of measures. PloS one, 10(5) (2015).

\section{Acknowledgment}

This work has received funding from the European Union's Horizon 2020 research and innovation programme under grant agreement No 769574. 


\section{Conflict of Interest}

The authors declare that they have no conflict of interest. 